\title{
The Detection of Hot Cores and Complex Organic Molecules in the Large Magellanic Cloud
}

\author{
Marta Sewiło $^{1,13}$ (1) , Remy Indebetouw ${ }^{2,3}$ (1) , Steven B. Charnley ${ }^{1}$ (i), Sarolta Zahorecz ${ }^{4,5}$ (i), Joana M. Oliveira ${ }^{6}$ (i), \\ Jacco Th. van Loon $^{6}$ (i) , Jacob L. Ward ${ }^{7}$, C.-H. Rosie Chen ${ }^{8}$ (iD), Jennifer Wiseman ${ }^{1}$ (i), Yasuo Fukui ${ }^{9}$, Akiko Kawamura ${ }^{10}$ (1), \\ Margaret Meixner ${ }^{11}$, Toshikazu Onishi $^{4}$ (i), and Peter Schilke ${ }^{12}$ (1) \\ ${ }^{1}$ NASA Goddard Space Flight Center, 8800 Greenbelt Road, Greenbelt, MD 20771, USA; marta.m.sewilo@nasa.gov \\ ${ }^{2}$ Department of Astronomy, University of Virginia, P.O. Box 400325, Charlottesville, VA 22904, USA \\ ${ }^{3}$ National Radio Astronomy Observatory, 520 Edgemont Road, Charlottesville, VA 22903, USA \\ ${ }^{4}$ Department of Physical Science, Graduate School of Science, Osaka Prefecture University, 1-1 Gakuen-cho, Naka-ku, Sakai, Osaka 599-8531, Japan \\ ${ }^{5}$ Chile Observatory, National Astronomical Observatory of Japan, National Institutes of Natural Science, 2-21-1 Osawa, Mitaka, Tokyo 181-8588, Japan \\ ${ }^{6}$ Lennard-Jones Laboratories, Keele University, Staffordshire ST5 5BG, UK \\ ${ }^{7}$ Astronomisches Rechen-Institut, Zentrum für Astronomie der Universität Heidelberg, Mönchhofstr. 12-14, D-69120 Heidelberg Germany \\ ${ }_{9}^{8}$ Max-Planck-Institut für Radioastronomie, Auf dem Hügel 69, D-53121 Bonn, Germany \\ ${ }^{9}$ School of Science, Nagoya University, Furo-cho, Chikusa-ku, Nagoya 464-8602, Japan \\ ${ }^{10}$ National Astronomical Observatory of Japan, 2-21-1 Osawa, Mitaka, Tokyo 181-8588, Japan \\ ${ }^{11}$ Space Telescope Science Institute, 3700 San Martin Drive, Baltimore, MD 21218, USA \\ ${ }^{12}$ I. Physikalisches Institut der Universität zu Köln, Zülpicher Str. 77, D-50937 Köln, Germany \\ Received 2017 September 12; revised 2017 December 6; accepted 2017 December 8; published 2018 January 30
}

\begin{abstract}
We report the first extragalactic detection of the complex organic molecules $(\mathrm{COMs})$ dimethyl ether $\left(\mathrm{CH}_{3} \mathrm{OCH}_{3}\right)$ and methyl formate $\left(\mathrm{CH}_{3} \mathrm{OCHO}\right)$ with the Atacama Large Millimeter/submillimeter Array (ALMA). These COMs, together with their parent species methanol $\left(\mathrm{CH}_{3} \mathrm{OH}\right)$, were detected toward two $1.3 \mathrm{~mm}$ continuum sources in the N 113 star-forming region in the low-metallicity Large Magellanic Cloud (LMC). Rotational temperatures $\left(T_{\text {rot }} \sim 130 \mathrm{~K}\right)$ and total column densities $\left(N_{\text {rot }} \sim 10^{16} \mathrm{~cm}^{-2}\right)$ have been calculated for each source based on multiple transitions of $\mathrm{CH}_{3} \mathrm{OH}$. We present the ALMA molecular emission maps for COMs and measured abundances for all detected species. The physical and chemical properties of two sources with COMs detection, and the association with $\mathrm{H}_{2} \mathrm{O}$ and $\mathrm{OH}$ maser emission, indicate that they are hot cores. The fractional abundances of COMs scaled by a factor of 2.5 to account for the lower metallicity in the LMC are comparable to those found at the lower end of the range in Galactic hot cores. Our results have important implications for studies of organic chemistry at higher redshift.
\end{abstract}

Key words: astrochemistry - galaxies: star formation - Magellanic Clouds - stars: protostars

\section{Introduction}

Complex organic molecules (COMs, $\geqslant 6$ atoms including carbon; e.g., $\mathrm{CH}_{3} \mathrm{OH}, \mathrm{CH}_{3} \mathrm{OCH}_{3}, \mathrm{CH}_{3} \mathrm{OCHO}$ ) are widespread in the Milky Way galaxy where they have primarily been found in the environments of young protostars-hot cores and hot corinos (e.g., Herbst \& van Dishoeck 2009). Hot cores are compact $(D \lesssim 0.1 \mathrm{pc})$, hot $\left(T_{\text {kin }}>100 \mathrm{~K}\right)$, dense $\left(n_{\mathrm{H}}>\right.$ $10^{6-7} \mathrm{~cm}^{-3}$ ) sources (e.g., Kurtz et al. 2000) where ice mantles have recently been removed from dust grains, either by thermal evaporation or sputtering in shock waves. Interstellar COMs may be a chemical link to the prebiotic molecules that were involved in the processes leading to the origin of life (Ehrenfreund \& Charnley 2000).

The observed hot core molecules may have their origin in the chemistry of the cold prestellar phase, either through formation in gas-phase reactions followed by freeze-out on the dust, or as products of reactions on the icy mantles of the dust grains (Brown et al. 1988). Grain-surface production could involve atom addition reactions on cold dust (e.g., Charnley \& Rodgers 2008) or radical reactions on warm dust during the thermal warm up of the hot core (Garrod \& Herbst 2006). Alternatively, COMs and other molecules may be formed in subsequent gas-phase reactions in the hot gas (e.g., Charnley et al. 1992).

\footnotetext{
${ }^{13}$ NASA Postdoctoral Program Fellow.
}

In galaxies with subsolar metallicities, questions remain as to the formation efficiency of COMs (e.g., Acharyya \& Herbst 2015; Shimonishi et al. 2016a). Apart from the lower elemental abundances of gaseous $\mathrm{C}, \mathrm{O}$, and $\mathrm{N}$ atoms, low metallicity leads to less shielding, greater penetration of UV photons into dense gas, and consequently warmer dust grains (e.g., van Loon et al. 2010; Oliveira et al. 2011). All of these factors inhibit the formation and survival of COMs.

The Large Magellanic Cloud (LMC) is a nearby dwarf galaxy $(50.0 \pm 1.1 \mathrm{kpc}$; Pietrzyński et al. 2013) that presents the opportunity to study COM chemistry in a low-metallicity environment $\left(Z_{\mathrm{LMC}} \sim 0.3-0.5 Z_{\odot} ;\right.$ Westerlund 1997) with higher UV fluxes and lower cosmic-ray ionization rates than the Milky Way (e.g., Abdo et al. 2010). Shimonishi et al. (2016b) have claimed a hot core detection toward the LMC young stellar object (YSO) ST11 based on the derived physical conditions and the presence of simple molecules connected to hot gas chemistry (e.g., $\mathrm{SO}_{2}$ and its isotopologues). Neither methanol or COMs were detected.

Nishimura et al. (2016a) observed seven molecular clouds in the LMC at low spatial resolution $(\sim 8 \mathrm{pc})$ and also failed to detect methanol or any COMs. Several transitions of methanol have previously been reported in two star-forming regions in the LMC (N 159W and N 113; Heikkilä et al. 1999; Wang et al. 2009), both included in the Nishimura et al. (2016a) sample, and a few $\mathrm{CH}_{3} \mathrm{OH}$ masers are reported in the literature 


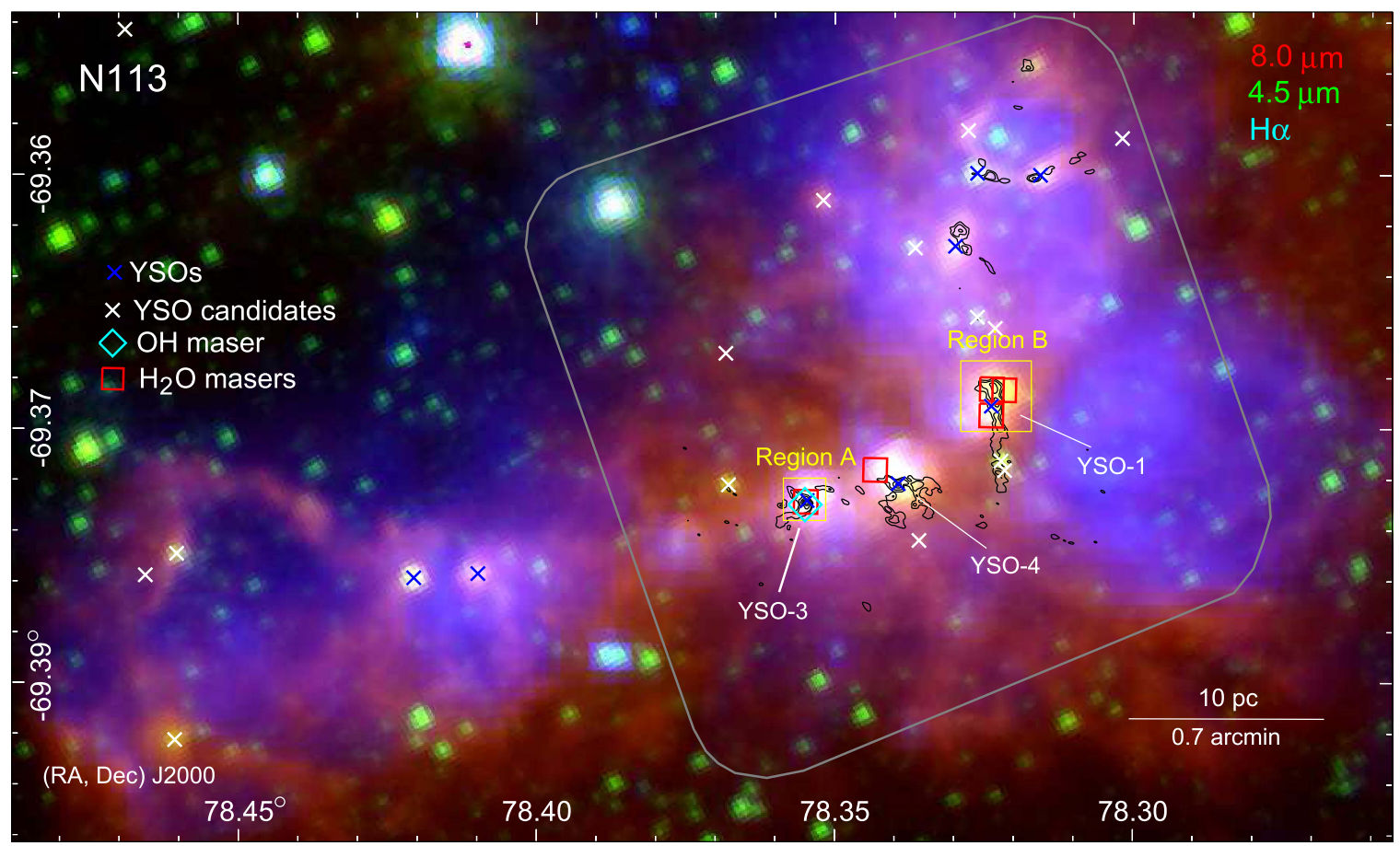

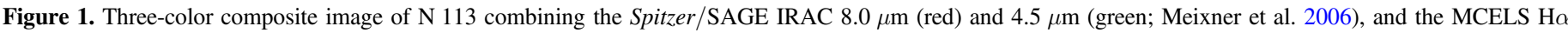

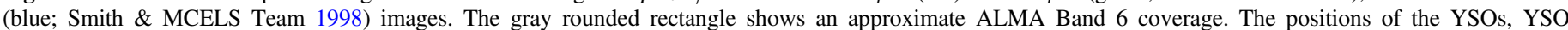

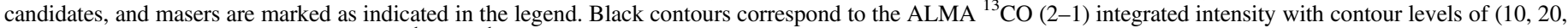

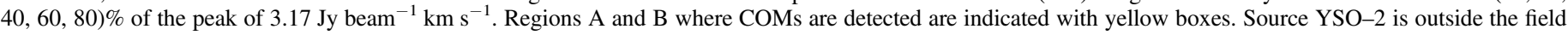
of view.

(e.g., Sinclair et al. 1992; Green et al. 2008). The existing studies indicate that the abundance of $\mathrm{CH}_{3} \mathrm{OH}$ in the LMC is very low, with potentially important general implications for COM formation in low-metallicity galaxies and at high redshift.

The sources observed by Nishimura et al. (2016a) contain large abundances of $\mathrm{CCH}$, a good photodissociation region (PDR) tracer, with the highest $\mathrm{CCH}$ column density found in $\mathrm{N} 113$. This suggests that the formation of COMs in the LMC and other low-metallicity galaxies (e.g., IC10; Nishimura et al. 2016b) could be inhibited by the presence of extensive PDRs (Hollenbach \& Tielens 1999). Apart from destruction by photolysis, warm dust ( $\gtrsim 20 \mathrm{~K}$ ) leads to inefficient $\mathrm{H}$ atom sticking and $\mathrm{CO}$ hydrogenation to methanol on grain surfaces (e.g., Watanabe \& Kouchi 2008).

In this Letter, we report the first extragalactic detection of the COMs dimethyl ether $\left(\mathrm{CH}_{3} \mathrm{OCH}_{3}\right)$ and methyl formate $\left(\mathrm{CH}_{3} \mathrm{OCHO}\right)$ in the $\mathrm{N} 113$ region of the LMC. We present the Atacama Large Millimeter/submillimeter Array (ALMA) molecular emission maps and measured abundances of the COMs. The relationship to Galactic hot cores and the implications for extragalactic organic chemistry are briefly discussed.

\section{The N113 Star-forming Region}

LHA 120-N 113 (N 113; Henize 1956) is one of the most prominent star formation regions in the LMC. It contains one of the most massive $\left(\sim 10^{5} M_{\odot}\right)$ and richest giant molecular clouds (GMCs) in the LMC. Its peak CO (1-0) brightness temperature of $\sim 8.1 \mathrm{~K}$ is the highest in the MAGMA survey with the Mopra telescope (half-power beam width, HPBW $\sim 45^{\prime \prime}$; Wong et al. 2011).

The dense molecular gas in the N 113 GMC is clumpy with substructures that are directly revealed by the high volume density tracers $\mathrm{HCO}^{+}$and $\mathrm{HCN}$ (e.g., Seale et al. 2012). N 113 hosts the largest number of $\mathrm{H}_{2} \mathrm{O}$ and $\mathrm{OH}$ masers and the brightest $\mathrm{H}_{2} \mathrm{O}$ maser in the entire LMC (e.g., Whiteoak \& Gardner 1986; Green et al. 2008; Ellingsen et al. 2010), all located within $25^{\prime \prime}$ ( $\sim 6 \mathrm{pc}$ ) from the GMC's CO (1-0) peak.

Current star formation activity in $\mathrm{N} 113$ appears concentrated in the central part of the GMC as indicated by point-like midinfrared emission, maser sources, and compact $\mathrm{H}$ II regions, superposed on an extended emission component and aligned in a northwest-southeast direction (see Figure 1). The gas and dust in this central region are compressed by a complex structure of ionized gas bubbles (prominent in the $\mathrm{H} \alpha$ images) created by massive stars in several young clusters (Oliveira et al. 2006). Based on the Spitzer Space Telescope and the Herschel Space Observatory data, confirmed and candidate Stage 0 -II YSOs down to $\sim 3 M_{\odot}$ were identified (e.g., Sewiło et al. 2010; Carlson et al. 2012).

At Spitzer and Herschel wavelengths (3.6-500 $\mu \mathrm{m}$ with $2^{\prime \prime}-38^{\prime \prime}$ resolution), N 113 is dominated by emission from three massive (30-40 $M_{\odot}$; Ward et al. 2016) YSOs associated with highdensity molecular clumps, radio continuum emission, and masers: YSO-1 (051317.69-692225.0; Gruendl \& Chu 2009), YSO-3 (051325.09-692245.1), and YSO-4 (051321.43-692241.5); see Figure 1. Our Herschel spectroscopic data (e.g., [O I], [O III], [C II]) reveal distinct physical conditions around each of these nearby sources (J. M. Oliveira et al., in preparation). $K$-band observations obtained with VLT/SINFONI with 0 ." 1 resolution and a field of view of $\sim 3^{\prime \prime}$ resolved YSO-3 and YSO-4 into a cluster of multiple components and revealed diverse characteristics of resolved sources (Ward et al. 2016). N 113 is a complex environment where we find clumps $(\sim 1 \mathrm{pc})$ and cores $(\sim 0.1-0.2 \mathrm{pc})$ in a range of environments (e.g., strongly influenced by the stellar winds in the west and more quiescent in the east) and at a range of evolutionary stages. 


\section{ALMA Observations of $\mathbf{N} 113$}

$\mathrm{N} 113$ was observed with ALMA in Band 6 as part of project 2015.1.01388.S. The spectral setup observed ${ }^{12} \mathrm{CO}(2-1)$, ${ }^{13} \mathrm{CO}(2-1)$, and $\mathrm{C}^{18} \mathrm{O}(2-1)$ in narrow windows and additional $2 \mathrm{GHz}$ windows centered on 231.7 and $216.9 \mathrm{GHz}$.

The data were obtained with the $12 \mathrm{~m}$ array on 2016 March 10 and June 16 , for a total of 13.1 minutes per mosaic pointing over most of the map, on baselines from 15 to $704 \mathrm{~m}$. It was also observed 19 times with the $7 \mathrm{~m}$ Atacama Compact Array (ACA) array between 2015 November 2 and December 17, for a total of 247 minutes per mosaic pointing. The data were calibrated with versions 4.7 through 5.0 of the ALMA pipeline in Common Astronomy Software Applications (CASA; McMullin et al. 2007). Amplitude was calibrated using Uranus, J0538-4405, J0519-4546, and Callisto (different calibrators on different dates). The bandpass was calibrated using J0538-4405, J0522-3627, J0854+2006, J0006-0623, and time-varying gain using J0529-7245 (12 m array) and J0440-6952 (7 m array).

Sensitivity of $0.1 \mathrm{mJy}$ per 0 "! $87 \times 0$ " $54(\sim 0.21 \times 0.13 \mathrm{pc})$ beam was achieved in the continuum. Continuum was subtracted in the $u v$ domain from each line spectral window, and the $7 \mathrm{~m}$ and $12 \mathrm{~m}$ data were simultaneously imaged and deconvolved interactively. Sensitivity of $3.8 \mathrm{mJy}$ per 0 "' $98 \times 0$ " $62(\sim 0.24 \times$ $0.15 \mathrm{pc}$ ) beam was achieved in the $216.9 \mathrm{GHz}$ cube with $1.09 \mathrm{MHz}\left(1.5 \mathrm{~km} \mathrm{~s}^{-1}\right)$ channels, and $2.9 \mathrm{mJy}$ per 0 " $88 \times 0$ ". 58 $(\sim 0.21 \times 0.14 \mathrm{pc})$ beam in $2.0 \mathrm{MHz}\left(2.6 \mathrm{~km} \mathrm{~s}^{-1}\right)$ channels at $231.7 \mathrm{GHz}$.

The distribution of our project's main targeted bright lines over the entire N 113 region will be discussed in a future publication, as the scope of this Letter is the complex organic molecules located in two small regions in the center of $\mathrm{N} 113$ (Regions A and B in Figure 1).

\section{Results \\ 4.1. $1.3 \mathrm{~mm}$ Continuum Emission}

We have identified multiple $1.3 \mathrm{~mm}$ continuum sources in both Regions A and B (see Figure 2). We have assigned identification numbers to all of the continuum peaks that are associated with the molecular or $\mathrm{H} 30 \alpha$ line emission in the order from the brightest to faintest. There is a number of the continuum peaks that are less significant and/or not associated with the molecular or $\mathrm{H} 30 \alpha$ line emission that will be discussed in a follow-up paper. COMs have been detected toward sources $\mathrm{A} 1$ and B3. Both sources are associated with $\mathrm{H}_{2} \mathrm{O}$ masers and A1 also with an $\mathrm{OH}$ maser (within the positional uncertainties). Only one SINFONI $K$-band source (N113-YSO01) has been detected at $1.3 \mathrm{~mm}$ (B2).

There is some evidence for a small difference in the evolutionary stage between A1 and B3. B3 is brighter than A1 in DCN, which indicates that it is younger. The DCN/HCN ratio will drop rapidly with time in hot gas, irrespective of the origin of DCN in the gas phase or on ice. The abundance of all deuterated species decreases with time (Fontani et al. 2011); in hot cores, they are fossils of the earlier, colder evolutionary phases. The more mature nature of A1 is supported by an association with the $\mathrm{OH}$ maser.

We measured the continuum surface brightness for A1 and B 3 in the continuum image centered at $224.6 \mathrm{GHz}$ by fitting the sources with Gaussians, as well as measuring the value of the brightest pixel. The weighted average of the fitted and manually measured peaks $(0.58 \pm 0.09 \mathrm{~K}$ and $0.29 \pm 0.02 \mathrm{~K}$ for A1 and B3, respectively) was used to calculate the dust column density. The dust optical depth was calculated by assuming that the dust temperature equals the fitted $\mathrm{CH}_{3} \mathrm{OH}$ kinetic temperature (see Section 4.2). The largest source of uncertainty is the choice of dust emissivity used to calculate column density from optical depth. We use values calculated specifically for the LMC by Galliano et al. (2011), albeit on somewhat larger scales $(\geqslant 10 \mathrm{pc}): N\left(\mathrm{H}_{2}\right) / \tau_{\text {dust }}=1.8 \times$ $10^{26} \mathrm{~cm}^{-2}$. If dust has coagulated on the small scales probed by ALMA, the dust emissivity could be higher, hydrogen column densities lower, and organic molecule abundances higher than we quote here. We determined $N\left(\mathrm{H}_{2}\right)$ of $(8.0 \pm$ $1.2) \times 10^{23} \mathrm{~cm}^{-2}$ and $(7.0 \pm 0.9) \times 10^{23} \mathrm{~cm}^{-2}$ for A1 and B3, respectively. The source diameters are about the beam size; thus, under the assumption of spherical symmetry, these column densities correspond to the number densities of $\sim 1.6 \times$ $10^{6} \mathrm{~cm}^{-3}$ and $\sim 1.4 \times 10^{6} \mathrm{~cm}^{-3}$, respectively.

\subsection{Spectral Line Analysis}

The spectra for $\mathrm{A} 1$ and $\mathrm{B} 3$ are shown in Figure 3 . We detected $\mathrm{COMs}\left(\mathrm{CH}_{3} \mathrm{OH}, \mathrm{CH}_{3} \mathrm{OCHO}\right.$, and $\left.\mathrm{CH}_{3} \mathrm{OCH}_{3}\right)$, sulfurbearing molecules $\left(\mathrm{SO}_{2}, \mathrm{H}_{2} \mathrm{~S}, \mathrm{SiO}, \mathrm{OCS},{ }^{13} \mathrm{CS}\right.$, and $\left.{ }^{33} \mathrm{SO}\right)$, $c-\mathrm{C}_{3} \mathrm{H}_{2}$, and DCN (see Table 1). We have carried out the line identification with the MADCUBAIJ software (Martín et al. 2011; Rivilla et al. 2016), which uses the JPL ${ }^{14}$ and CDMS ${ }^{15}$ molecular line databases. This software provides theoretical synthetic spectra of the different molecules under local thermodynamic equilibrium (LTE) conditions, taking into account the individual opacity of each line. To identify a molecule, the detectable lines in the observed spectra predicted by the LTE analysis must be present, with the relative intensities of the different transitions consistent with the LTE analysis.

We used the results of the rotational diagram analysis (e.g., Goldsmith \& Langer 1999) of $\mathrm{CH}_{3} \mathrm{OH}$ as the initial parameters for the spectral line modeling using MADCUBAIJ. We utilized six $\mathrm{CH}_{3} \mathrm{OH}$ transitions with a range of upper energy levels $\left(E_{\mathrm{u}} \sim 56-508 \mathrm{~K}\right.$; see Figure 4$)$. This analysis assumes the gas is in LTE and the lines are optically thin. We obtained the rotational temperatures and total column densities $\left(T_{\text {rot }}, N_{\text {rot }}\right)$ of $\left(134 \pm 6 \mathrm{~K}, 1.6 \pm 0.1 \times 10^{16} \mathrm{~cm}^{-2}\right)$ and $(131 \pm 15 \mathrm{~K}, 6.4 \pm$ $0.8 \times 10^{15} \mathrm{~cm}^{-2}$ ) for A1 and B3, respectively. The synthetic spectra corresponding to these parameters are shown in Figure 3. The uncertainties for the calculated temperature and column density values are the formal fit uncertainties from the least squares fits performed by MADCUBAIJ.

Assuming that all molecular species observed toward A1/B3 are located in the same region as methanol, we estimate their column densities using the same $T_{\text {rot }}$ as for methanol (see Table 1). To investigate how the results would change if we assumed a different value of $T_{\text {rot }}$, we calculated column densities for $T_{\text {rot }}$ of $100,150,200$, and $250 \mathrm{~K}$. In general, column densities increase gradually with increasing $T_{\text {rot }}$, and they are not larger than a factor of $\sim 2$ than those listed in Table 1 for any molecule at $T_{\text {rot }}=250 \mathrm{~K}$. At $100 \mathrm{~K}$, column densities are in general up to $\sim 20 \%$ lower.

A non-detection of $\mathrm{CH}_{3} \mathrm{OH}$ in $\mathrm{N} 113$ by Nishimura et al.'s (2016a) single-dish observations can be explained by the fact that they covered Region B only. Region B harbors the fainter

\footnotetext{
14 http://spec.jpl.nasa.gov/

15 http://www.astro.uni-koeln.de/cdms
} 

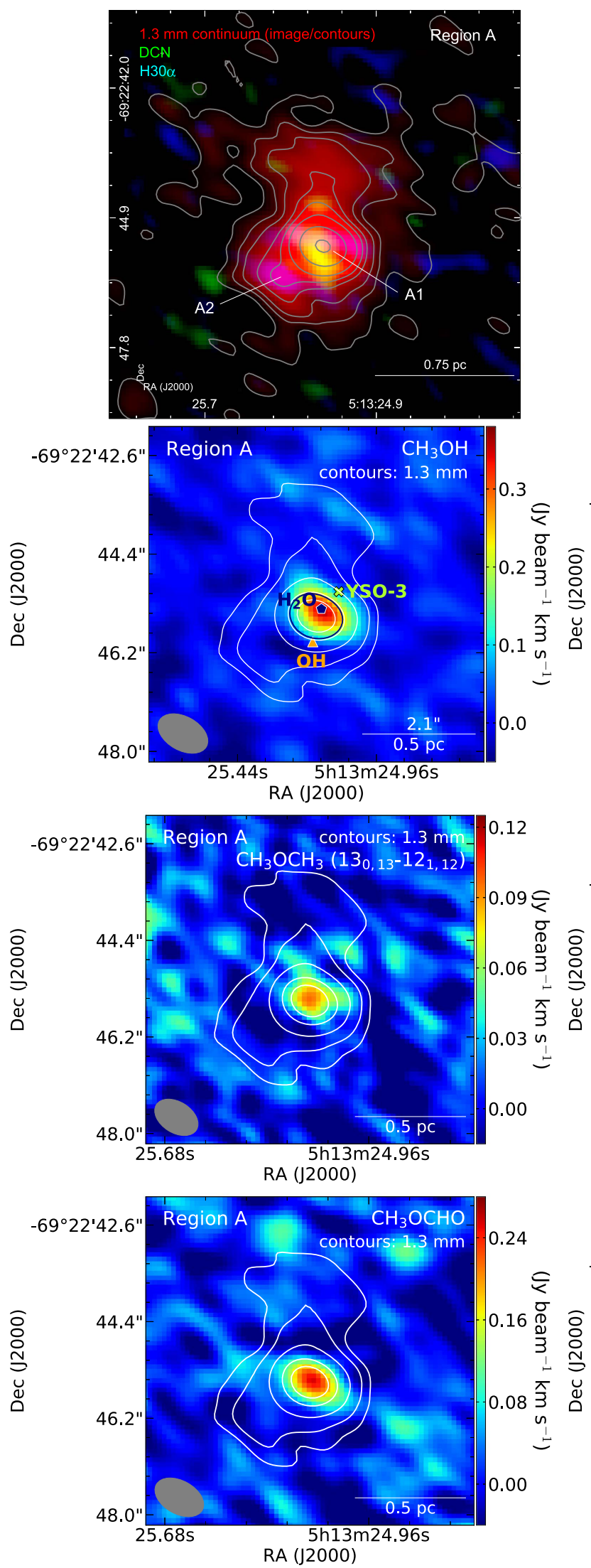
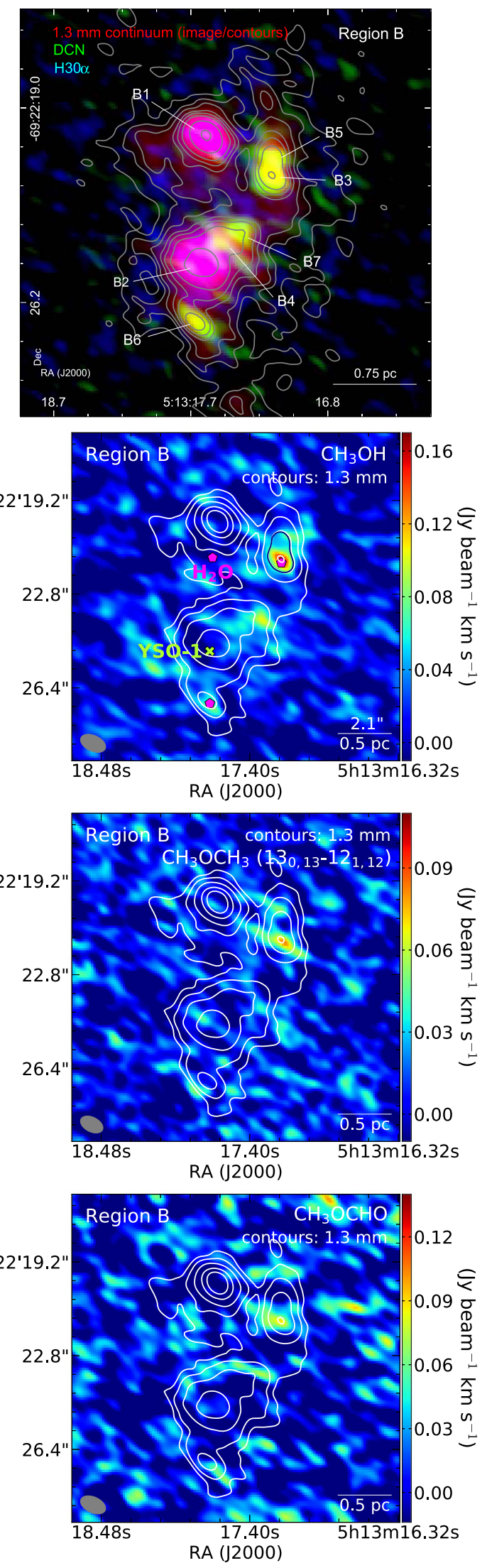

Figure 2. Top panels: the three-color mosaics combining the $1.3 \mathrm{~mm}$ continuum (red), DCN (green), and H30 $\alpha$ (blue) images for Regions A (left) and B (right). The $1.3 \mathrm{~mm}$ continuum contour levels are $(3,6,9,15,20,30,60,120) \times 0.1 \mathrm{mJy}^{-}$beam ${ }^{-1}(1 \sigma)$. Second row: the $\mathrm{CH}_{3} \mathrm{OH}$ integrated intensity image for Regions A and B with selected $1.3 \mathrm{~mm}$ continuum contours overlaid for reference. The $\mathrm{CH}_{3} \mathrm{OH}$ images were made using the channels corresponding to all $\mathrm{CH}_{3} \mathrm{OH}$ transitions in the $216.9 \mathrm{GHz}$ spectral window. The positions of the $\mathrm{H}_{2} \mathrm{O}$ (pentagons) and $\mathrm{OH}$ (triangle) masers and Spitzer YSOs (" $\times$ ") are indicated. The areas enclosed by navy contours were used to extract the spectra shown in Figure 3. The $\mathrm{CH}_{3} \mathrm{OCH}_{3}\left(13_{0,13}-12_{1,12}\right)$ and $\mathrm{CH}_{3} \mathrm{OCHO}$ (integrated over all transitions) images for Regions A and $\mathrm{B}$ are shown in the third and fourth rows. 

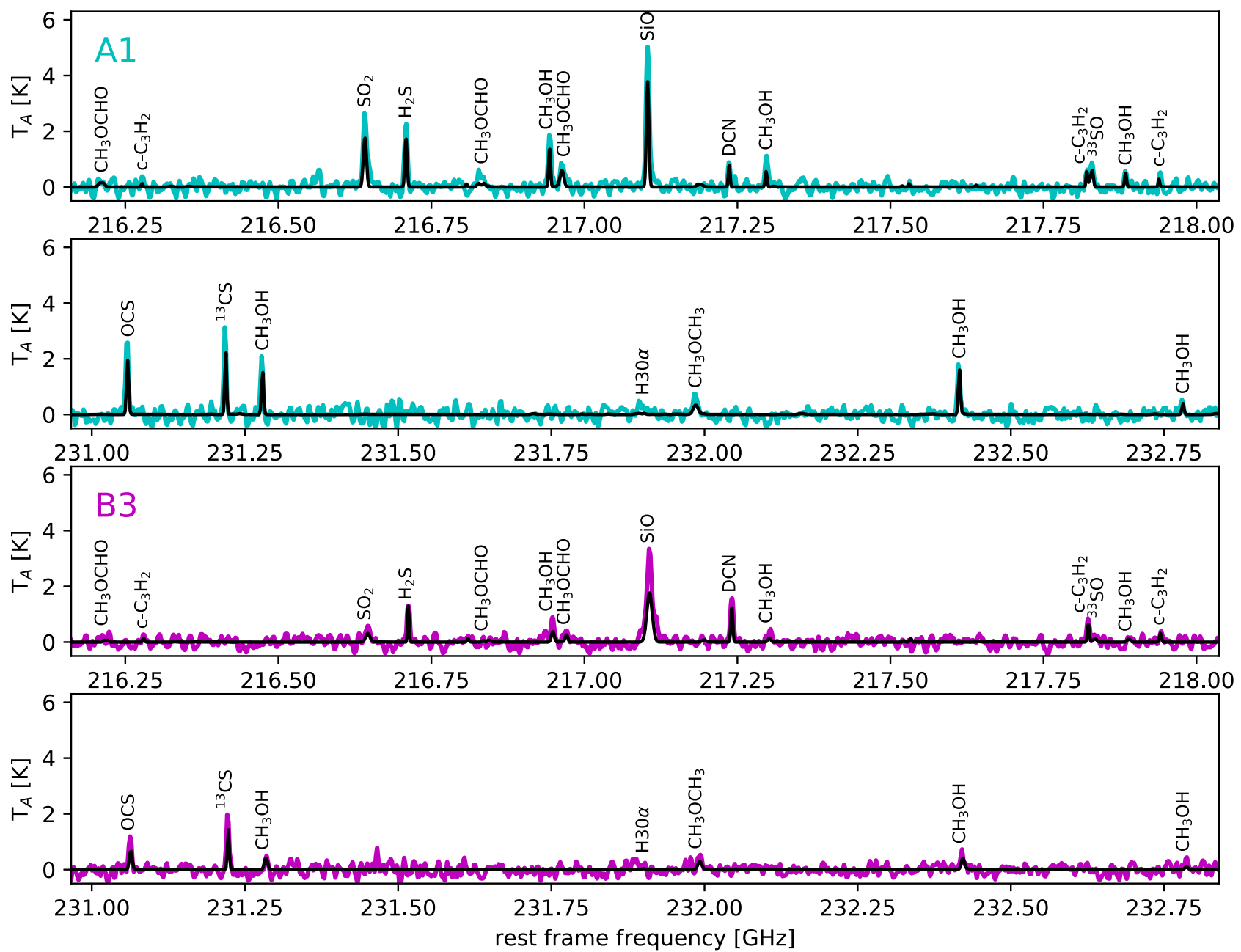

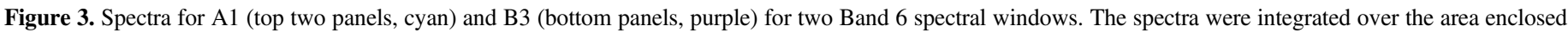

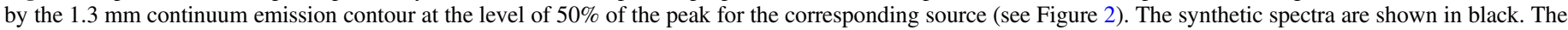
molecular transitions listed in Table 1 are indicated.

and smaller of the two sources with $\mathrm{CH}_{3} \mathrm{OH}$ detection, suffering larger effects from beam dilution. The single-dish observations of Wang et al. (2009) that detected methanol in N 113 had a similar sensitivity; however, they covered both Regions $\mathrm{A}$ and $\mathrm{B}$. The $\mathrm{CH}_{3} \mathrm{OH}$ column density estimated by Wang et al. $\left(2009 ; \sim 10^{13} \mathrm{~cm}^{-2}\right)$ is lower than that based on the ALMA data, likely the effect of the beam dilution. Only with ALMA can the clumpy structure of methanol be revealed.

\section{Discussion}

Both A1 and B3 resemble classic hot cores-dense condensations of molecular gas surrounding the massive star formation sites. They are compact $(D \sim 0.17 \mathrm{pc})$ and hot $\left(T_{\text {rot }} \sim 130 \mathrm{~K}\right)$, as evidenced by the high excitation lines of methanol with rotation temperatures similar to Galactic hot cores at the lower end of the $T_{\text {rot }}$ range (e.g., Kurtz et al. 2000). A1 and B3 are slightly larger than "typical" Galactic hot cores, but their number densities and column densities are consistent with those of known hot cores, they are hot enough to release ice mantles, they show emission from COMs, and they are associated with masers; all of these properties support the classification of A1 and B3 as hot cores.

Some fainter methanol emission is detected in other locations in Region B (see Figure 2); however, it is unclear whether that traces physically distinct sources or shocked lobes of the outflows.

The detection of $\mathrm{SiO}$ demonstrates that shock sputtering of dust and ice may be as important as thermal evaporation for initiating the chemical evolution in these hot cores (e.g., Charnley \& Kaufman 2000; Viti et al. 2001). A SiH 4 (i.e., nonshock) origin for $\mathrm{SiO}$ is possible (Mackay 1996), but it requires that hot cores contain abundances of $\mathrm{O}_{2}$ that are significantly above the observed upper limits obtained with Herschel (e.g., Liseau et al. 2012; Y1ldiz et al. 2013).

The detection of $\mathrm{CH}_{3} \mathrm{OH}$ indicates that the dust was once cold enough for $\mathrm{CO}$ hydrogenation to proceed and the observed DCN is probably a frozen gas-phase remnant of this cold cloud chemistry (Brown \& Millar 1989).

Using the $\mathrm{CH}_{3} \mathrm{OH}$ and $\mathrm{H}_{2}$ column densities derived above, we calculate the fractional abundance of $\mathrm{CH}_{3} \mathrm{OH}$ of $(2.0 \pm 0.3) \times 10^{-8}$ and $(9.1 \pm 1.7) \times 10^{-9}$ for $\mathrm{A} 1$ and $\mathrm{B} 3$, respectively. These $\mathrm{CH}_{3} \mathrm{OH}$ fractional abundances are over an order of magnitude larger than an upper limit estimated for the candidate hot core ST11 by Shimonishi et al. (2016b). The $\left(\mathrm{CH}_{3} \mathrm{OCH}_{3}, \mathrm{CH}_{3} \mathrm{OCHO}\right)$ fractional abundances are $(2.2 \pm 0.7$, $1.4 \pm 0.4) \times 10^{-9}$ for A1 and $(1.7 \pm 0.7,<0.5) \times 10^{-9}$ for B3. The $\left(\mathrm{CH}_{3} \mathrm{OCH}_{3}, \mathrm{CH}_{3} \mathrm{OCHO}\right)$ abundances with respect to $\mathrm{CH}_{3} \mathrm{OH}$ are $(0.11 \pm 0.03,0.07 \pm 0.01)$ and $(0.19 \pm 0.07$, $<0.05)$ for A1 and B3, respectively. 
Table 1

Molecular Line Transitions Detected toward A1 and B3

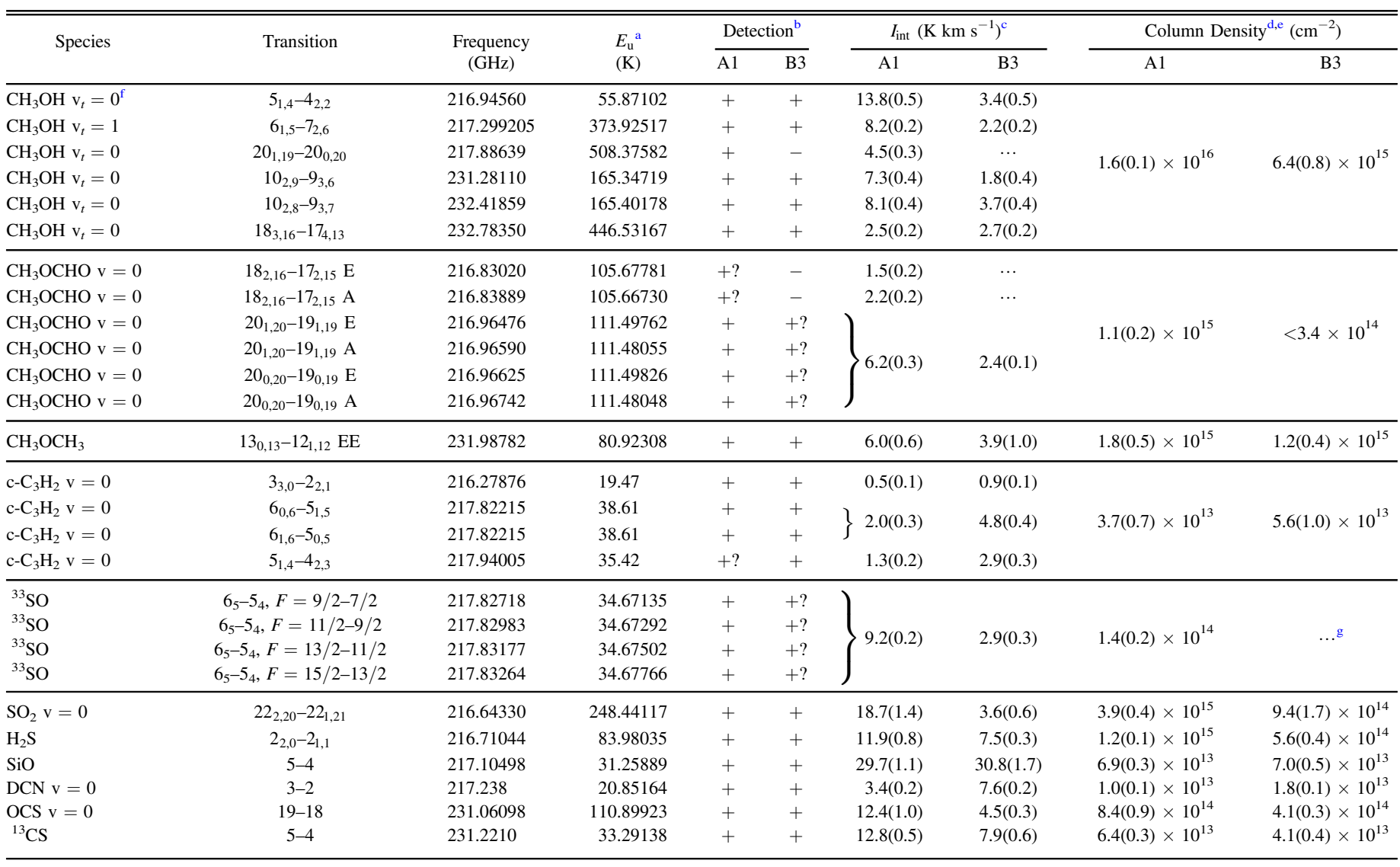

Notes.

${ }^{\mathrm{a}} E_{u}$ is the upper level energy of the transition.

b The symbols in these columns indicate a detection (“+”), a tentative detection (“+?"), or a non-detection (“-”) of a given molecular line transition.

${ }^{\mathrm{c}} I_{\text {int }}$ is the integrated line intensity.

${ }^{d}$ The $\mathrm{CH}_{3} \mathrm{OH}$ column densities and temperatures were derived based on modeling of multiple $\mathrm{CH}_{3} \mathrm{OH}$ transitions (see the text). Column densities of other molecules were estimated assuming the same temperature as for methanol.

${ }^{\mathrm{e}}$ For molecules with multiple transitions, all the transitions were used to estimate a total column density.

${ }^{\mathrm{f}}$ A typical line width for the strongest methanol line is $\sim 5 \mathrm{~km} \mathrm{~s}^{-1}$ for A1 and $\sim 8 \mathrm{~km} \mathrm{~s}^{-1}$ for B3.

${ }^{\mathrm{g}}{ }^{33} \mathrm{SO}$ lines for B3 are too faint to get a good fit.

The abundances of COMs detected in N 113 are comparable to those found at the lower end of the range in Galactic hot cores (e.g., Herbst \& van Dishoeck 2009; Taquet et al. 2016), when scaled by a factor of 2.5 to account for the lower metallicity in the LMC (assuming $Z_{\mathrm{LMC}}=0.4 Z_{\odot}$ ). Thus, the chemistry of COMs detected in N 113 is similar to that of the Galaxy, indicating that regions where they exist are likely shielded from UV radiation (see Cuadrado et al. 2017). Both grain reactions on warm dust and post-desorption ion-molecule chemistry could form $\mathrm{CH}_{3} \mathrm{OCH}_{3}$ and $\mathrm{CH}_{3} \mathrm{OCHO}$ (Garrod \& Herbst 2006; Taquet et al. 2016).

We have also detected several S-bearing molecules in both cores. In $\mathrm{A} 1$ and $\mathrm{B} 3$, the $\mathrm{H}_{2} \mathrm{~S} / \mathrm{CH}_{3} \mathrm{OH}$ ratios are respectively $0.075 \pm 0.008$ and $0.088 \pm 0.013$, whereas the $\mathrm{OCS} / \mathrm{CH}_{3} \mathrm{OH}$ ratios are $0.053 \pm 0.007$ and $0.064 \pm 0.009$. These ratios are almost indistinguishable between $\mathrm{A} 1$ and $\mathrm{B} 3$, supporting the idea that these molecules were formed in ices during the N113 prestellar phase. OCS is known to be present in interstellar ices (Boogert et al. 2015) and, although $\mathrm{H}_{2} \mathrm{~S}$ has not yet been

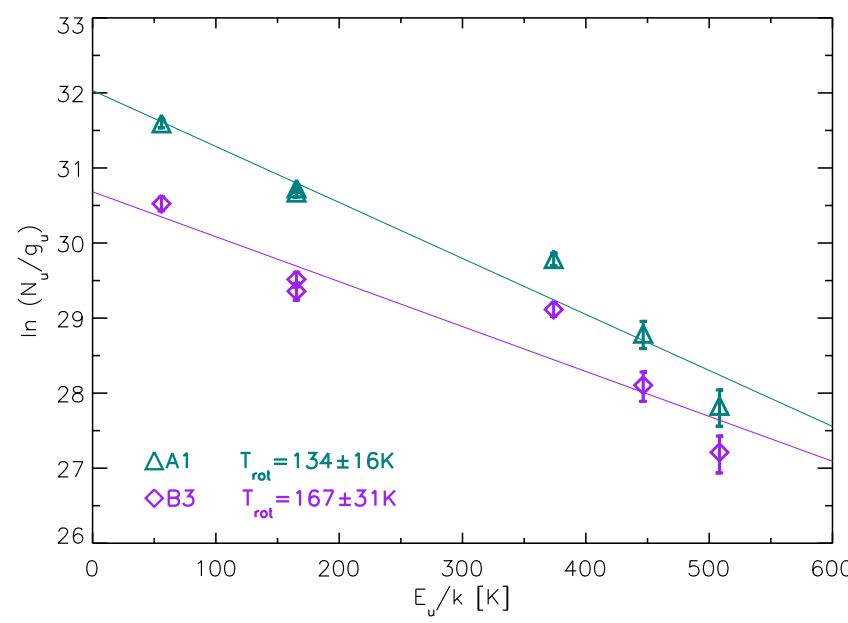

Figure 4. Rotational diagram of $\mathrm{CH}_{3} \mathrm{OH}$ detected toward $\mathrm{A} 1$ and $\mathrm{B} 3$. The results of the rotational diagram analysis were used as the initial parameters for the MADCUBAIJ modeling. 
confirmed, cold grain-surface chemistry, involving reactions of $\mathrm{S}$ atoms with atomic hydrogen and $\mathrm{CO}$ (or oxidation of $\mathrm{CS}$ ), is likely to be the origin of these molecules in the N113 cores.

$\mathrm{SO}$ and $\mathrm{SO}_{2}$ could also originate from ice chemistry (they are detected in cometary ices; Calmonte et al. 2016), but they may also form in post-shock gas that is enriched in $\mathrm{OH}$ through neutral-neutral reactions (Hartquist et al. 1980). Alternatively, chemical models show that all the S-bearing molecules, including $\mathrm{SO}$ and $\mathrm{SO}_{2}$, could be formed in post-evaporation gas-phase reactions starting from evaporated ices containing $\mathrm{H}_{2} \mathrm{~S}$ (Charnley 1997). In this case, comparison of calculated and observed $\mathrm{SO} / \mathrm{SO}_{2}, \mathrm{H}_{2} \mathrm{~S} / \mathrm{SO}_{2}$, and $\mathrm{H}_{2} \mathrm{~S} / \mathrm{SO}$ ratios could be used as a chemical clock for the age of the core. We will explore this issue in a future publication.

Finally, $c-\mathrm{C}_{3} \mathrm{H}_{2}$ could either be formed by ion-molecule chemistry in the cool ambient medium or, like $\mathrm{CCH}$, be in a $\mathrm{PDR}$ region, where it may be produced in the photodestruction of larger refractory organic (PAH) molecules (Guzmán et al. 2014).

\section{Conclusions}

We see the effective formation of COMs under subsolar metallicity conditions in two hot cores discovered in the LMC. The COMs observed in the N113 hot cores could either originate from grain-surface chemistry or in post-desorption gas chemistry. Of the S-bearing molecules detected, it is likely that $\mathrm{H}_{2} \mathrm{~S}$ and OCS formed on grains; $\mathrm{SO}$ and $\mathrm{SO}_{2}$ also could have originated from ice chemistry or formed in gaseous neutral reactions. The presence of $\mathrm{SiO}$ indicates that shock chemistry may also be playing a role. Future observations of LMC cores and detailed chemical modeling will be necessary to determine the relative contribution from each process. Studying the chemistry of the interstellar medium as a function of metallicity is important to understand the chemical evolution of the universe.

We thank the anonymous referee for insightful comments and suggestions that helped us improve the Letter. The work of M.S. was supported by an appointment to the NASA Postdoctoral Program at the Goddard Space Flight Center, administered by Universities Space Research Association under contract with NASA. S.C. acknowledges the support from the NASA's Emerging Worlds Program. S.Z. was supported by NAOJ ALMA Scientific Research grant number 2016-03B. J.L.W. acknowledges support from the German Research Council (SFB 881 "The Milky Way System", subproject P1). M.M. was supported by NSF grant 1312902. The National Radio Astronomy Observatory is a facility of the National Science Foundation operated under cooperative agreement by Associated Universities, Inc. This Letter makes use of the following ALMA data: ADS/JAO. ALMA\#2015.1.01388.S. ALMA is a partnership of ESO (representing its member states), NSF (USA) and NINS (Japan), together with NRC (Canada), NSC and ASIAA (Taiwan), and KASI (Republic of Korea), in cooperation with the Republic of Chile. The Joint ALMA Observatory is operated by ESO, AUI/NRAO and NAOJ.

\section{ORCID iDs}

Marta Sewiło (1) https://orcid.org/0000-0003-2248-6032 Remy Indebetouw (i) https://orcid.org/0000-0002-4663-6827

Steven B. Charnley (ib https://orcid.org/0000-0001-6752-5109 Sarolta Zahorecz (i) https://orcid.org/0000-0001-6149-1278

Joana M. Oliveira (iD https://orcid.org/0000-0002-0861-7094

Jacco Th. van Loon (iD https://orcid.org/0000-0002-1272-3017 C.-H. Rosie Chen (ib https://orcid.org/0000-0002-3925-9365 Jennifer Wiseman (iD https://orcid.org/0000-0002-1143-6710 Akiko Kawamura (iD https://orcid.org/0000-0001-7813-0380 Toshikazu Onishi (iD https://orcid.org/0000-0001-7826-3837 Peter Schilke (iD https://orcid.org/0000-0003-2141-5689

\section{References}

Abdo, A. A., Ackermann, M., Ajello, M., et al. 2010, A\&A, 512, A7 Acharyya, K., \& Herbst, E. 2015, ApJ, 812, 142

Boogert, A. C. A., Gerakines, P. A., \& Whittet, D. C. B. 2015, ARA\&A, 53, 541

Brown, P. D., Charnley, S. B., \& Millar, T. J. 1988, MNRAS, 231, 409

Brown, P. D., \& Millar, T. J. 1989, MNRAS, 237, 661

Calmonte, U., Altwegg, K., Balsiger, H., et al. 2016, MNRAS, 462, 253

Carlson, L. R., Sewiło, M., Meixner, M., Romita, K. A., \& Lawton, B. 2012, A\&A, 542, A66

Charnley, S. B. 1997, ApJ, 481, 396

Charnley, S. B., \& Kaufman, M. J. 2000, ApJ, 529, 111

Charnley, S. B., \& Rodgers, S. D. 2008, SSRv, 138, 59

Charnley, S. B., Tielens, A. G. G. M., \& Millar, T. J. 1992, ApJ, 399, 71

Cuadrado, S., Goicoechea, J. R., Cernicharo, J., et al. 2017, A\&A, 603, A124

Ehrenfreund, P., \& Charnley, S. B. 2000, ARA\&A, 38, 427

Ellingsen, S. P., Breen, S. L., Caswell, J. L., Quinn, L. J., \& Fuller, G. A. 2010 , MNRAS, 404, 779

Fontani, F., Palau, A., Caselli, P., et al. 2011, A\&A, 529, 7

Galliano, F., Hony, S., Bernard, J.-P., et al. 2011, A\&A, 536, A88

Garrod, R. T., \& Herbst, E. 2006, A\&A, 457, 927

Goldsmith, P. F., \& Langer, W. D. 1999, ApJ, 517, 209

Green, J. A., Caswell, J. L., Fuller, G. A., et al. 2008, MNRAS, 385, 948

Gruendl, R. A., \& Chu, Y. 2009, ApJS, 184, 172

Guzmán, V. V., Pety, J., Gratier, P., et al. 2014, FaDi, 168, 103

Hartquist, T. W., Dalgarno, A., \& Oppenheimer, M. 1980, ApJ, 236, 182

Heikkilä, A., Johansson, L. E. B., \& Olofsson, H. 1999, A\&A, 344, 817

Henize, K. G. 1956, ApJS, 2, 315

Herbst, E., \& van Dishoeck, E. F. 2009, ARA\&A, 47, 427

Hollenbach, D. J., \& Tielens, A. G. G. M. 1999, RvMP, 71, 173

Kurtz, S., Cesaroni, R., Churchwell, E., Hofner, P., \& Walmsley, C. M. 2000, in Protostars and Planets IV, ed. V. Mannings, A. P Boss, \& S. S. Russell (Tucson, AZ: Univ. Arizona Press), 299

Liseau, R., Goldsmith, P. F., Larsson, B., et al. 2012, A\&A, 541, 73

Mackay, D. D. S. 1996, MNRAS, 278, 62

Martín, S., Krips, M., Martín-Pintado, J., et al. 2011, A\&A, 527, A36

McMullin, J. P., Waters, B., Schiebel, D., Young, W., \& Golap, K. 2007, in ASP Conf. Ser. 376, Astronomical Data Analysis Software and Systems XVI, ed. R. A. Shaw, F. Hill, \& D. J. Bell (San Francisco, CA: ASP), 127

Meixner, M., Gordon, K. D., Indebetouw, R., et al. 2006, AJ, 132, 2268

Nishimura, Y., Shimonishi, T., Watanabe, Y., et al. 2016a, ApJ, 818, 161

Nishimura, Y., Shimonishi, T., Watanabe, Y., et al. 2016b, ApJ, 829, 94

Oliveira, J. M., van Loon, J. T., Stanimirovíc, S., \& Zijlstra, A. A. 2006, MNRAS, 372, 1509

Oliveira, J. M., van Loon, J. Th., Sloan, G. C., et al. 2011, MNRAS, 411, 36 Pietrzyński, G., Graczyk, D., Gieren, W., et al. 2013, Natur, 495, 76 Rivilla, V. M., Fontani, F., Beltrán, M. T., et al. 2016, ApJ, 826, 161 Seale, J. P., Looney, L. W., Wong, T., et al. 2012, ApJ, 751, 42

Sewiło, M., Indebetouw, R., Carlson, L. R., et al. 2010, A\&A, 518, L73

Shimonishi, T., Dartois, E., Onaka, T., \& Boulanger, F. 2016a, A\&A, 585, A107

Shimonishi, T., Onaka, T., Kawamura, A., \& Aikawa, Y. 2016b, ApJ, 827, 72

Sinclair, M. W., Carrad, G. J., Caswell, J. L., Norris, R. P., \& Whiteoak, J. B. 1992, MNRAS, 256, 33 
Smith, R. C. \& MCELS Team 1998, PASA, 15, 163

Taquet, V., Wirström, E. S., \& Charnley, S. B. 2016, ApJ, 821, 46

van Loon, J. Th., Oliveira, J. M., Gordon, K. D., Sloan, G. C., \& Engelbracht, C. W. 2010, AJ, 139, 1553

Viti, S., Caselli, P., Hartquist, T. W., \& Williams, D. A. 2001, A\&A, 370, 1017

Wang, M., Chin, Y.-N., Henkel, C., Whiteoak, J. B., \& Cunningham, M. 2009, ApJ, 690, 580
Ward, J. L., Oliveira, J. M., van Loon, J. T., \& Sewiło, M. 2016, MNRAS, 455,2345

Watanabe, N., \& Kouchi, A. 2008, PrSS, 83, 439

Westerlund, B. E. 1997, The Magellanic Clouds (New York: Cambridge Univ. Press)

Whiteoak, J. B., \& Gardner, F. F. 1986, MNRAS, 222, 513

Wong, T., Hughes, A., Ott, J., et al. 2011, ApJS, 197, 16

Yıldız, U. A., Acharyya, K., Goldsmith, P. F., et al. 2013, A\&A, 558, 58 